\title{
Knowledge, attitude, practice, and pharmaceutical outcomes of type 2 diabetes mellitus self- management among patients in Makkah Region, Saudi Arabia
}

\begin{abstract}
Introduction: Type 2 diabetes mellitus (T2DM) is increasing worldwide and Saudi Arabia (SA). The diabetes clinics and hospital emergencies in Makkah region receive increased numbers of patients with T2DM complications, especially during Hajj and Umrah seasons. Enhancing self-management in patients with T2DM can improve their daily practice and reduce the risk of associated complications and their referrals towards hospital emergencies. Additionally, pharmaceutical intervention and drug compliance are essential for the management of T2DM complications. Self-management can be assessed by measuring the patient's knowledge, attitude, and practice (KAP) towards T2DM. This study aims to measure KAP levels of self-management of T2DM and the pharmaceutical outcomes among patients in Makkah region, SA.
\end{abstract}

Materials and methods: This is a cross-sectional study of patients with T2DM conducted for four months between January 2020-April 2020. Individuals completed a validated online administered questionnaire using the Survey Monkey website. Data collectors approached potential participants at locations including; governmental hospitals, leisure centers, and shopping malls. Descriptive analysis included frequency and percentage for categorical variables, and mean and standard deviation (SD) for continuous variables. In addition, the Chi-square test was used to test for association between two categorical variables.

Results: A total of 400 patients with T2DM with a mean age of 45 years (range 18-80 years) were surveyed. Eighty percent of participants reported a good knowledge of T2DM; however, they also reported a poor attitude $(65 \%)$ and practice $(62 \%)$. Participants of the old cohort group provided higher significant knowledge responses associated with the HbA1C measurement $(P<0.05)$ and the effect of T2DM on five organs in the body $(P<0.05)$ than the young cohort group. The most used antidiabetic medication classes among the patients were Metformin-Glucophage with $65 \%$ of the patients and Sitagliptin-Januvia with $45 \%$. In comparison, Liraglutide-Victoza was used with a lower percentage of only $25 \%$, and Ertugliflozin-Steglatro was the lowest antidiabetic used with only $15 \%$ of the patients.

Conclusion: The patient's good knowledge of T2DM disease was not reflected in the patient's attitude and practice towards T2DM. Therefore, the local health authorities need to implement health education and awareness of clinical pharmaceutical programs with the help of clinical pharmacists to improve patient's self-management towards T2DM. Furthermore, the local health authorities may increase the application of GLP-1 analogs, which is highly recommended for the treatment of T2DM that reduces the risk of T2DM complications.

Keywords: type 2 diabetes mellitus, knowledge, attitude, practice, pharmaceutical outcomes, Saudi Arabia, self-management, complications

\section{Introduction}

Type 2 diabetes mellitus (T2DM) is a clinical syndrome of disordered carbohydrates, fat, and protein metabolism. This condition results from an absolute or relative deficiency of insulin and a defect in insulin action known as insulin resistance (IR). ${ }^{1} \mathrm{~T} 2 \mathrm{DM}$ is characterized by chronic hyperglycemia and dyslipidemia, and it contributes to developing microvascular complications, cardiovascular disease (CVD), and renal disease. ${ }^{2}$ The Middle East has a high prevalence of T2DM due to the development and changes in diet patterns and rapid economic expansion. ${ }^{3}$ In Saudi Arabia (SA), the Ministry of Health $(\mathrm{MOH})$ stated that T2DM prevalence is about 2.5 million, and the rate is increasing. ${ }^{4}$
Volume 9 Issue 3 - 202I

\author{
Sharaf E Sharaf, ${ }^{1,2}$ Mohammad H Alsaedi,' \\ Alya'a B Bannani,' Motlaq N Al-Shammari,' \\ Torki A Alzahrani,' 'Mansur J Al-Otaibi,' \\ Shahd Y Alharbi,' Mohammed H Alharthy,' \\ Anmar A Alrefaei' \\ 'Department of Pharmaceutical Chemistry, College of Pharmacy, \\ Umm Al-Qura University, Saudi Arabia \\ ${ }^{2}$ Clinical research center, The Executive Administration of \\ Research and Innovation, King Abdullah Medical City (KAMC) in \\ Holy capital, Saudi Arabia
}

\section{Correspondence: Sharaf E Sharaf, Department of Pharmaceutical Chemistry, Ist floor, College of Pharmacy, Umm Al-Qura University, Makkah, Western Region, Saudi Arabia,} Email sesharaf@uqu.edu.sa

Received: May 24, 2021 | Published: June 07, 2021
Self-management through medication adherence, healthy lifestyle habits, behavior changes, and physical activity are essential for preventing and controlling T2DM complications. ${ }^{5-8}$ Therefore, one treatment approach for improving glycemic control in T2DM is enhancing patients' self-management with T2DM. ${ }^{9,10}$ In addition, the pharmaceutical intervention and patient's compliance are vital to improve glycemic control and manage the T2DM complications, and using the most recent approved antidiabetics classes is essential, especially in terms of replacing the previously identified ones with multi-antidiabetic actions such as Glucagon-like-peptide-1 analogs, i.e., Liraglutide (Victoza) $)^{11}$ and Sodium-glucose co-transporter-2 (SGLT2) inhibitors, i.e., Ertugliflozin (Steglatro). ${ }^{12}$ 
Makkah city is globally known as the Holy Capital regarding the Islamic religion, and millions of pilgrims and worshipers arrive monthly to Makkah during Hajj and Umrah seasons. ${ }^{13}$ Therefore, there is a noticed increase in patients with T2DM complications who are admitted to diabetes health clinics and hospital emergencies. ${ }^{4}$ Thus, high self-management levels regarding T2DM are highly required for the patients living in Makkah region. This will be reflected in their daily practice by maintaining and avoiding related T2DM complications. ${ }^{13}$ This will help the health authorities in Makkah region manage large numbers of patients, whether they were citizens or visitors from other regions in SA or overseas, who are attaining the Diabetes health clinics and hospital emergencies, especially during Hajj and Umrah seasons. ${ }^{13}$ The measurement of a patient's selfmanagement can be achieved by conducting knowledge, attitude, and practice (KAP) studies that assess the current related knowledge and awareness, which uncovers the weakest areas related to T2DM for potential improvement ${ }^{14}$ to the pharmaceutical outcomes. Previous studies were conducted in SA to determine the current KAP levels of T2DM in SA's central region ${ }^{15,16}$ and Hail region. ${ }^{17}$ Therefore, this study will measure the KAP of T2DM among patients in Makkah region, SA.

\section{Materials and methods}

\section{Ethical approval}

This study's documents were submitted to the institutional review board (IRB) at King Abdullah medical city (KAMC) in the Holy Capital, SA. The committee provided unconditional approval to this study, agreed to the use of verbal consent, and gave this study a registration number: (19-584).

\section{Sample size}

The sample size was calculated using Slovin's formula, with a population size of about 4.5 million, ${ }^{18}$ confidence interval (CI) 0.95 , and margin of error $5 \% .{ }^{19}$ Therefore, the required number of patients was 384 patients to achieve the required CI. The number of participants who consented to this study was $n=400$ to allow for potential dropouts.

\section{Study design}

This was a cross-sectional, questionnaire-based study conducted for four months between January 2020-April 2020. Potential participants were randomly approached in outpatient clinics at local hospitals, shopping centers, leisure centers, and recreational parks using a simple random sampling method. Data collectors were welltrained senior pharmacy students and qualified to conduct clinical research. The complete study aims and objectives were explained to the participants, and they were consented to participate in the study. The questionnaire was adopted from the Michigan Diabetes Research Training Centre (MDRTC) instrument surveys ${ }^{20}$ and two similar studies in SA conducted by Ibrahim Abougalambou et al., 2019 and by Aljofan et al., 2019..$^{15,17}$ Approval was received using the KAP diabetes survey instruments from MDRTC to edit, translate, and validate them to match our inclusion/exclusion criteria and research scope. The questionnaire was designed in English and then translated into Arabic, the local spoken language, by proficient speakers of both languages and revised to suit the general population. The correcting scheme for the KAP questions in each section was marked as one (1) point for correct answer and Zero (0) point for incorrect answers. ${ }^{15}$ The final score is calculated and demonstrated into two main categories for each KAP part as follows: ${ }^{15}$
- Good Knowledge: when the participant responds greater than or equal to seven, the mean score of knowledge questions. Poor Knowledge: when patients respond less than seven, the mean score of knowledge questions. ${ }^{15}$

- Good attitude: when the participant responds greater than or equal to six, the mean score of practice questions. Poor attitude: when patients respond less than six, the mean score of attitude questions. ${ }^{15}$

- Good practice: when the participant responds greater than or equal to six, the mean score of practice questions. Poor practice: when patients respond less than six, the mean score of practice questions. ${ }^{15}$

\section{Questionnaire}

The developed questionnaire was established to assess the participant's KAP regarding T2DM, its metabolic complications, risk factors, and management using online survey development cloud-based software (Survey Monkey). The questionnaire was presented online to the patients using electronic tablets (such as Apple iPad and Samsung Galaxy Tab) to be filled by patients with T2DM using the Survey Monkey website link. The questionnaire was divided into six main parts; the first included socio-demographic information, including gender, age, education level, and current employment. The second part concerned the patients' clinical history: duration of having T2DM, and family members with T2DM. The third to the fifth parts discussed T2DM knowledge, attitude, and practice, respectively ${ }^{15}$ which comprises questions related to T2DM disease, risk factors, complications, and compliance. The final part discussed the prevalence of the main six antidiabetic classes used for each patient among the most popular approved medications in the Saudi Pharmaceutical Health Sector, which are; Biguanides (i.e., Metformin-Glucophage-short acting) 1000mg twice daily, dipeptidyl peptidase-4 (DPP-4) inhibitors (i.e., Gliptins-Sitagliptin-Januvia) $100 \mathrm{mg}$ once daily, Sulfonylureas (i.e., Gliclazide-Diamicron) 30mg once daily, premixed 70/30 insulin (i.e., Humulin or Novomix 100 IU Vial $10 \mathrm{ml}$ ) long acting-once daily, GLP-1 analogs (i.e., LiraglutideVictoza) $1.8 \mathrm{mg}$ once daily, and SGLT-2 inhibitors (i.e., ErtugliflozinSteglatro) $5 \mathrm{mg}$ once daily.

For the questionnaire validation, a pilot sample was conducted using 25 randomly selected patients with T2DM. ${ }^{15}$ A total of 12 patients were requited from KAMC, and the remaining 13 were recruited from Al-Noor Specialist Hospital. The participants were informed of the study and consented to participate voluntarily. After that, the corrected version of the questionnaire from the pilot study was presented to a specialized committee of clinical professionals in the field at KAMC for final approval and opinion regarding the questionnaire's clinical and pharmaceutical content. After conducting a test-retest reliability test, good reliability was achieved. Finally, the final form of the questionnaire was used for this study.

\section{Study populations (inclusion/exclusion criteria)}

The selection criteria included male and female Saudi national patients living in Makkah region with T2DM between $\geq 18$ and $\leq 80$ years old. The exclusion criteria included non-Saudi patients, patients without T2DM, inability to consent, participants with medical background (all-health-related education). All data collected responses were downloaded from the Survey Monkey website and held on a secure server. 


\section{Statistical analysis}

The study was a complete case analysis. Participants who provided incomplete responses to the questionnaire were excluded. The data was collected from Survey Monkey's speared sheets to Microsoft excel for coding, then transferred to GraphPad Prism version 8.4.3 for statistical analysis. Descriptive statistics were used for demographic variables description, knowledge, attitude, and practice. A Chi-square test was used to detect significance between KAP and variables. Statistical significance was determined for a P-value of $<0.05$.

\section{Results}

\section{Demographic characteristic}

A total number of 420 questionnaires were collected voluntarily, of which 20 were excluded; most of them did not provide a complete response to the questionnaire, giving a response rate of $95 \%$. Thus, 400 questionnaires were included in the study. The participant's demographic characteristics are shown in Table 1. There were $\mathrm{n}=160$ males $(40 \%)$ and 240 females with T2DM. The majority of the participants were in the 41-80 years age group (old cohort group)
(73.5\%), and the remaining were in the $18-40$ years age group (young cohort group) (26.5\%). Most of the participants (78.5\%) received a university education, $12.5 \%$ completed High school education $(12.5 \%)$, and $9 \%$ had no formal educational qualifications. A total of $89 \%(n=386)$ of the participants were working, and most of the participants were having T2DM for more than five years $(63.5 \%)$ and had a family history of T2DM (87\%).

\section{Knowledge assessment}

The overall percentage of knowledge towards T2DM was good among the participants, with a total parentage of $80 \%$ (Figure 1). Figure 2 shows the total percentage distribution of knowledge among the participants. About $86 \%$ of the participants $(n=344)$ knew what T2DM; however, only $57 \%$ knew that food high in carbohydrates increases blood glucose levels (BGL). The association between the participant's knowledge and the participant's characteristics and educational background is shown in Table 2. Participants in the old cohort group scored significantly higher responses $(\mathrm{P}<0.05)$ in the knowledge of the period of $\mathrm{HbA} 1 \mathrm{C}$ measurement as well as linking with the effect of T2DM on five organs in the body (eyes, kidneys, foot ulcer, nerves, heart $)(\mathrm{P}<0.05)$ compared with younger participants.

Table I Socio-demographic characteristics and clinical history of T2DM among patients

\begin{tabular}{|c|c|}
\hline Variables & n (\%) \\
\hline \multicolumn{2}{|l|}{ Gender } \\
\hline Male & $160(40 \%)$ \\
\hline Female & $240(60 \%)$ \\
\hline \multicolumn{2}{|l|}{ Age } \\
\hline $18-40$ years & $106(26.5 \%)$ \\
\hline $4 \mid-80$ years & $294(73.5 \%)$ \\
\hline \multicolumn{2}{|l|}{ Education } \\
\hline None & $36(9 \%)$ \\
\hline High school & $50(12.5 \%)$ \\
\hline University & 314 (78.5\%) \\
\hline \multicolumn{2}{|c|}{ Employment } \\
\hline Working & 356 (89\%) \\
\hline Not working & 44 (I I\%) \\
\hline \multicolumn{2}{|c|}{ Duration of T2DM } \\
\hline$<5$ year & 146 (36.5\%) \\
\hline$>5$ years & $254(63.5 \%)$ \\
\hline \multicolumn{2}{|c|}{ Family history of T2DM } \\
\hline Yes & 348 (87\%) \\
\hline No & 52 (I3\%) \\
\hline
\end{tabular}

Data is represented as the number of participants and their percentage out of $n=400 ;$ T2DM, type 2 diabetes mellitus 


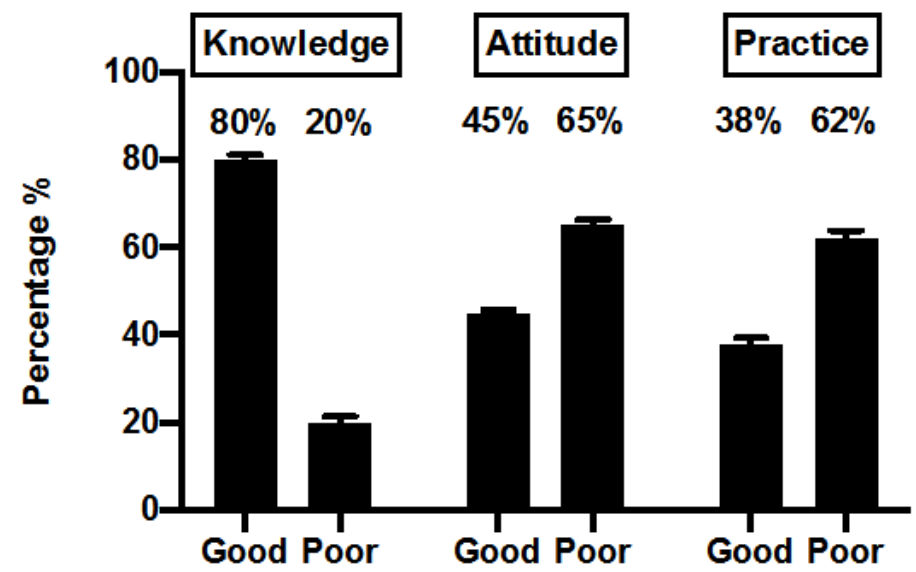

Figure I Total distribution of KAP of T2DM among participants.

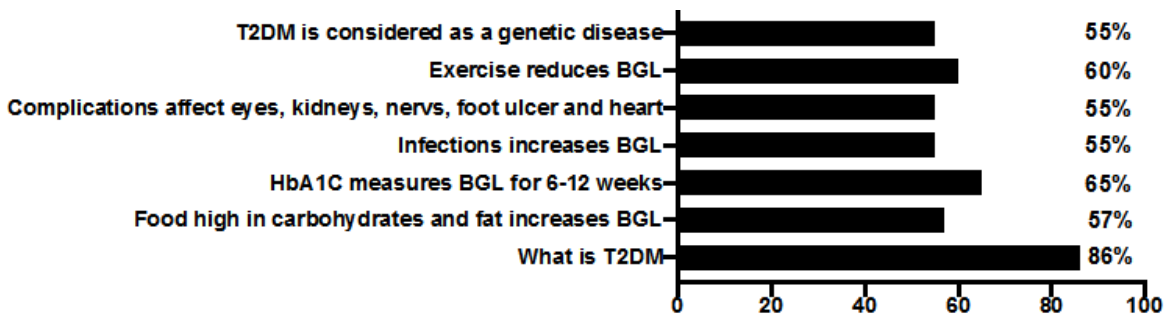

Figure 2 Frequency distribution of the correct knowledge responses.

T2DM, type 2 diabetes mellitus; BGL, blood glucose levels; HbAIC, hemoglobin AIC.

Table 2 The participant's correct knowledge responses associated with the participant's characteristics and educational background

\begin{tabular}{|c|c|c|c|c|c|c|c|c|c|c|c|}
\hline \multirow{3}{*}{$\begin{array}{l}\text { Knowledge } \\
\text { assessment } \\
\text { questions }\end{array}$} & \multicolumn{2}{|c|}{ Gender } & \multicolumn{2}{|l|}{ Age } & \multicolumn{3}{|c|}{ Educational background } & \multicolumn{2}{|c|}{ Employment } & \multicolumn{2}{|c|}{$\begin{array}{l}\text { Duration of } \\
\text { T2DM }\end{array}$} \\
\hline & Male & Female & $18-40$ & $41-70$ & None & High & University & Working & $\begin{array}{l}\text { Not } \\
\text { working }\end{array}$ & $\begin{array}{l}<5 \\
\text { Years }\end{array}$ & $\begin{array}{l}>5 \\
\text { Years }\end{array}$ \\
\hline & $\begin{array}{l}n=160 \\
(\%)\end{array}$ & $\begin{array}{l}n=240 \\
(\%)\end{array}$ & $\begin{array}{l}n=106 \\
(\%)\end{array}$ & $\begin{array}{l}n=294 \\
(\%)\end{array}$ & $\begin{array}{l}n=36 \\
(\%)\end{array}$ & $\begin{array}{l}n=50 \\
(\%)\end{array}$ & $n=3 \mid 4(\%)$ & $\begin{array}{l}n=356 \\
(\%)\end{array}$ & $\begin{array}{l}n=44 \\
(\%)\end{array}$ & $\begin{array}{l}n=\mid 46 \\
(\%)\end{array}$ & $\begin{array}{l}n=254 \\
(\%)\end{array}$ \\
\hline $\begin{array}{l}\text { What is } \\
\text { T2DM? }\end{array}$ & 85 & 86 & 30 & $92 *$ & 82 & 80 & 85 & 93 & 89 & 32 & $92 *$ \\
\hline $\begin{array}{l}\text { Food high in } \\
\text { carbohydrates } \\
\text { and fat } \\
\text { increases BGL? }\end{array}$ & 45 & 57 & 30 & $87 *$ & 35 & 55 & 60 & 75 & 80 & 32 & $85^{*}$ \\
\hline $\begin{array}{l}\text { HbAIC } \\
\text { measures } \\
\text { BGL for 6-I } 2 \\
\text { weeks? }\end{array}$ & 55 & 69 & 30 & $83^{*}$ & 55 & 65 & 50 & 90 & 70 & 35 & $87^{*}$ \\
\hline $\begin{array}{l}\text { Do infections } \\
\text { increase BGL? }\end{array}$ & 40 & 50 & 55 & 86 & 35 & 30 & 65 & 65 & 45 & 50 & 75 \\
\hline $\begin{array}{l}\text { Diabetes } \\
\text { complications } \\
\text { affect eyes, } \\
\text { kidneys, nerves, } \\
\text { foot ulcer, and } \\
\text { heart }\end{array}$ & 50 & 60 & 30 & $89 *$ & 40 & 60 & 75 & 80 & 75 & 35 & $85^{*}$ \\
\hline $\begin{array}{l}\text { Does exercise } \\
\text { reduce BGL? }\end{array}$ & 65 & 75 & 75 & 86 & 40 & 55 & 65 & 75 & 82 & 75 & 85 \\
\hline $\begin{array}{l}\text { T2DM is } \\
\text { considered } \\
\text { a genetic } \\
\text { disease? }\end{array}$ & 30 & 45 & 45 & 40 & 35 & 30 & $85^{*}$ & 55 & 40 & 45 & 55 \\
\hline
\end{tabular}

Data is represented as the correct response percentage out of the total number in each column. ${ }^{*}$, significant responses by Chi-square test with $\mathrm{P}$-values < 0.05 ; T2DM, type 2 diabetes mellitus; BGL, blood glucose levels 
Furthermore, participants in the old cohort group and those with T2DM for more than five years scored significantly higher responses $(\mathrm{P}<0.05)$ in the knowledge of increased BGL due to infections compared with younger participants who had T2DM less than five years. Furthermore, participants who received a university education scored significantly higher responses $(\mathrm{P}<0.05)$ and considered T2DM a partly genetic disease than the remaining participants. A total of $65 \%(n=260)$ of the participants knew that T2DM complications are associated with five main organs in the body as the following; $45 \%$ knew that T2DM complications are associated with the kidneys and nerves diseases, $40 \%$ thought that it is associated with eye disease, and 35\% listed foot ulcer and heart diseases (Figure 3).

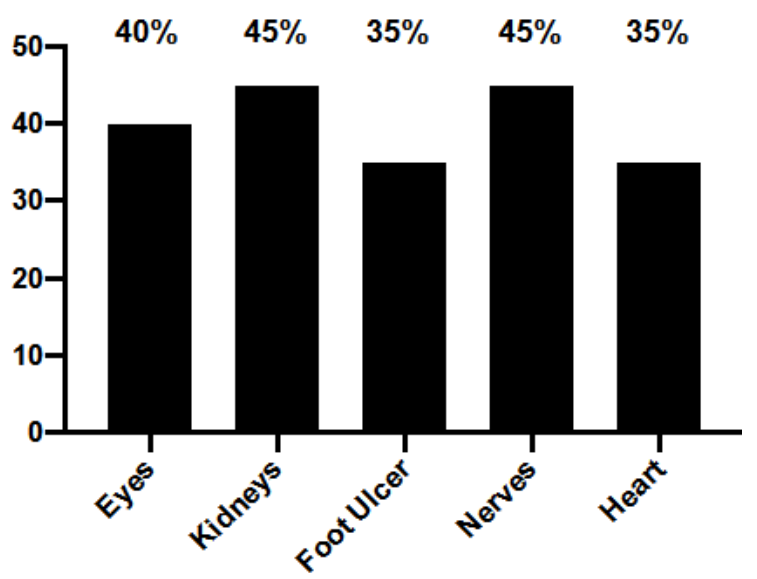

Figure 3 Frequency distribution of the long-term effects of T2DM complications on the main organs.

\section{Attitude assessment}

This study's participants reported a poor overall percentage of attitude towards T2DM of about $65 \%$ (Figure 1). The total attitude distribution of the participants was shown in Figure 4. This study did not detect any significant associations between the participant's attitudes towards T2DM and the participant's characteristic variables, education level, education, and employment level. Moreover, 295 participants $(73.8 \%)$ believed that the administration of alternative and herbal medicine without antidiabetic prescribed medication would maintain regular BGL. A total of 190 participants (47.5\%) believed that antidiabetic medication's long-term use is harmful. Surprisingly, $30 \%(\mathrm{n}=120)$ of the participants thought that T2DM is a temporary disease; 35\% (140) believed that antidiabetic medication could be immediately stooped once diabetes is controlled. Furthermore, 55\% $(n=220)$ of participants believed that proper foot care would prevent diabetic foot ulcers, and about half of the participants (50\%) believed that they should know more about T2DM self-management to improve practice (Figure 4).

\section{Practice assessment}

The major practice percentage among the participants was poor (62\%) regarding T2DM (Figure 1). The total practice percentage distribution of the participants is shown in Figure 5. Among the 400 participants, $200(50 \%)$ were reported to have checked their BGL regularly with a minimum of one time every year. Approximately $40 \%$ of the participants (160) claimed that they follow a suitable diet plan to maintain regular BGL, and 35\% (140) claimed that they are applying for an exercise program and keeping themselves physically active to maintain regular BGL. Unfortunately, 240 participants (60\%) reported that they suffer from T2DM complications, whether minor or significant. However, 160 of the participants $(40 \%)$ claimed that they attended health education centers to increase their knowledge of T2DM, and $220(55 \%)$ claimed that they had adopted lifestyle changes and welling to maintain regular BGL (Figure 5). No correlations were found between practice responses towards T2DM and all participants' variables.

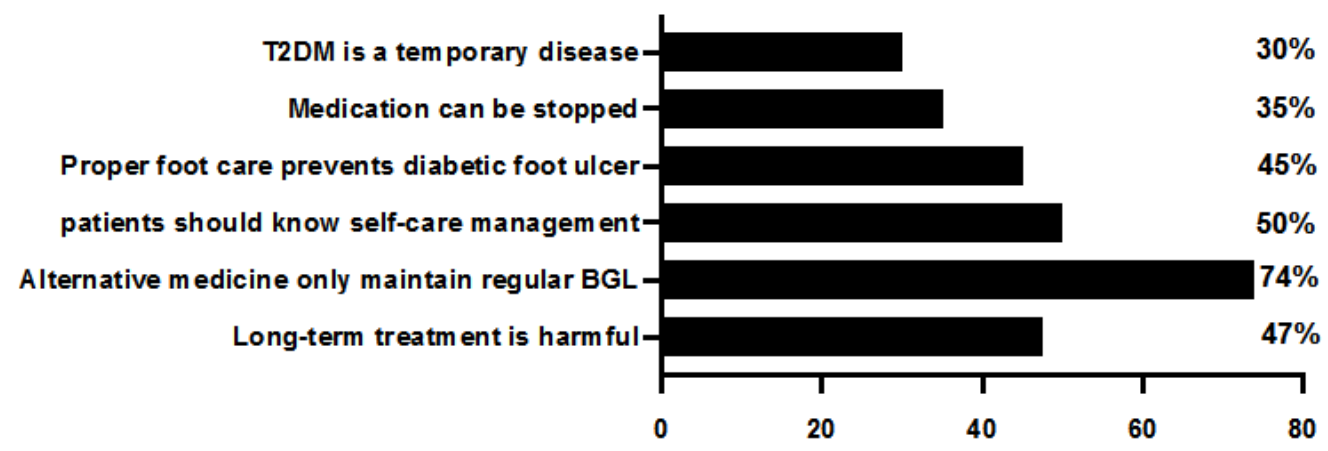

Figure 4 Frequency distribution of the correct attitude responses.

T2DM, type 2 diabetes mellitus; BGL, blood glucose levels.

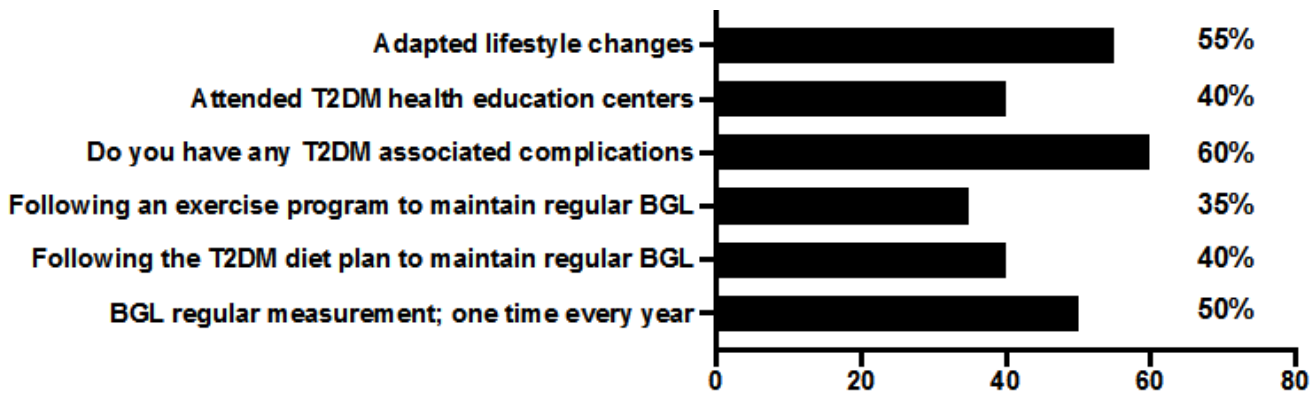

Figure 5 Frequency distribution of the correct practice responses.

T2DM, type 2 diabetes mellitus; BGL, blood glucose levels. 


\section{Pharmaceutical outcomes}

Figure 6 shows the prevalence of the main antidiabetic classes among the patients. Biguanides (i.e., Metformin-Glucophage) was the most class used $(65 \%)(n=260)$. The second most class used medication was DPP-4 inhibitors (i.e., Sitagliptin-Januvia) with
$45 \%(\mathrm{n}=180)$. The remining used classes were; Sulfonylureas (i.e., Gliclazide-Diamicron) with $36 \%(\mathrm{n}=144)$, premixed $70 / 30$ insulin (i.e., Humulin or Novomix $100 \mathrm{IU}$ Vial $10 \mathrm{ml}$ ) with $35 \%(\mathrm{n}=140)$, GLP-1 analogues (i.e., Liraglutide) with $25 \%(\mathrm{n}=100)$, and SGLT-2 inhibitors (i.e., Ertugliflozin- Steglatro) with the lowest percentage of $15 \%(n=60)$.

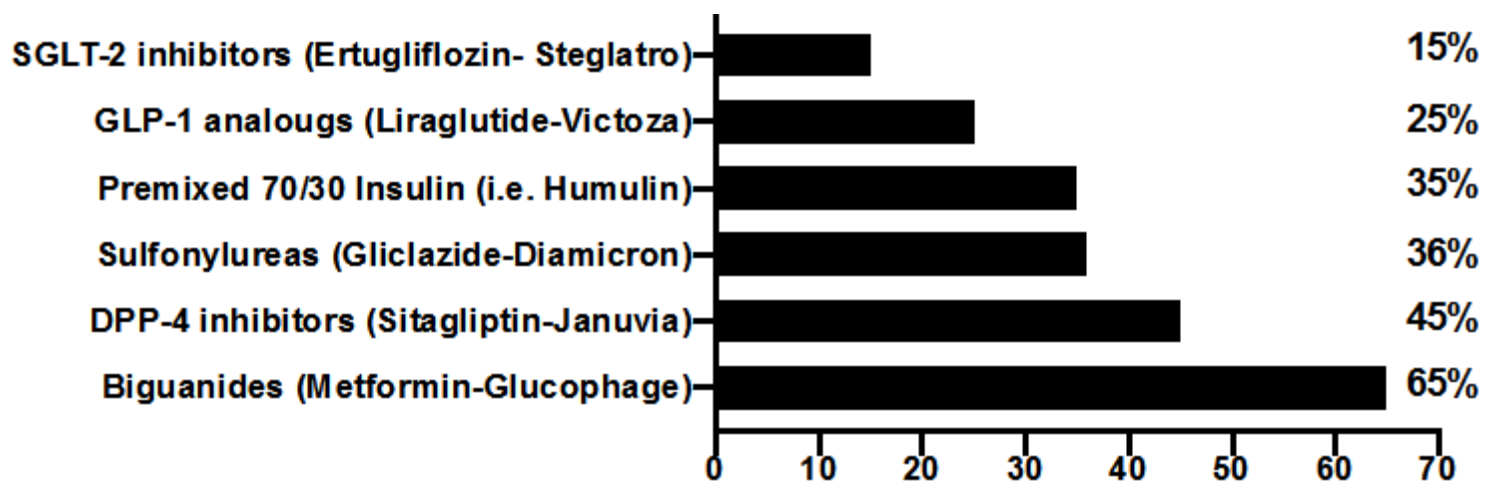

Figure 6 Prevalence of the main antidiabetic classes used among the patients.

SGLT-2, sodium-glucose transport protein 2; DPP-4, dipeptidyl peptidase-4; GLP-I, glucagon-like-peptide-I.

\section{Discussion}

Many recent studies have shown that improving the KAP of T2DM among patients is directly associated with their improvement in selfmanagement of T2DM and leads to better control of the disease. ${ }^{21-25} \mathrm{In}$ addition, American Diabetes Association (ADA) has also emphasized the importance of enhancing self-management to improve T2DM practice, compliance and reduce chronic complications. ${ }^{26}$ In general, participants of this study showed a high percentage of knowledge T2DM, which was similar to studies conducted in SA. ${ }^{15,17,27}$ This suggests that patients with T2DM in Saudi society have good Knowledge of T2DM, and the health authorities may not face significant obstacles during the application of further prevention and awareness plans.

The participants were better informed of the main organs affected by T2DM, similar to the previous similar studies conducted in SA. ${ }^{15,17,27}$ This could be explained as they experienced complications with similar organs themselves or observed them in fellow patients. In addition, they knew that a diet high in carbohydrates and lipids increases BGL, and regular exercise maintains normal BGL, which were similar in these similar studies. ${ }^{15,17,27}$ The higher percentage of educated participants in this study could explain these patient's responses. The participant's attitude towards T2DM was poor, which were similar study conducted by Alqahtani et al., ${ }^{27}$ and the results were the opposite in another study conducted by Ibrahim Abougalambou et al. ${ }^{15}$ This could be explained by sampling variability due to different sampling locations. Nevertheless, the participants believed that using alternative and herbal medicines without regularly prescribed medication could maintain regular BGL, similar to previous studies. ${ }^{15,27}$ This could indicate that patients need an education session that emphasizes the effects of alternative and herbal medication on T2DM and how to be used along with prescribed medication for the management and prevention strategies of T2DM.

The participants believed that they could stop medication after controlling their T2DM, and T2DM is a temporary disease that disappears once it is maintained. Similar findings were found in the study conducted by Aljofan et al. ${ }^{17}$ This indicates that the patients could face more T2DM complications as their glycemic control will not be maintained adequately based on their current attitude. Furthermore, the participants believed that long-term treatment with diabetes medication is harmful to the body, similar to these studies. ${ }^{17,27}$ This indicates that the awareness of T2DM medication in our participants meets expectations. The participants believed that T2DM selfmanagement is vital to improve their daily practice and compliance and reduce T2DM associated complications. This is a good indication that our participants are ready to look after themselves to reduce their T2DM complications, which is proven in many previous studies. ${ }^{9,23,28}$

The participant's practice towards T2DM was poor, similar in these studies. ${ }^{15,27}$ The participants measured BGL regularly every year, which was identical in the study conducted by Aljofan et al., ${ }^{15}$ and opposite results were also found in Alqahtani et al. ${ }^{27}$ This could indicate the importance of increasing the application of T2DM screening programs to detect patients' early diagnosis, preventing potential complications, and improving the patient's quality of life.

Most of the participants did not follow a diet plan, and a few of them conducted an exercise program to maintain regular BGL, which similar in these studies. ${ }^{15,27}$ In addition, most participants did not attend education health programs about T2DM in health care centers. However, almost half claimed to adopt lifestyle changes to improve their T2DM daily practice, which was similar in the study conducted by Aljofan et al. ${ }^{17}$ This is one reason that justifies the participant's poor attitude and practice levels as the diabetes health care centers provide education programs to enhance all KAP levels and educate the patients at all aspects of T2DM management.

This study shows that Metformin and Sitagliptin were the most used antidiabetics among the patients with T2DM; these results were similar to the survey conducted by AlKhamees et al. ${ }^{29}$ The other remaining antidiabetics classes, mainly the GLP-1 analogs such as Liraglutide (Victoza) and SGLT-2 inhibitors such as Ertugliflozin (Steglatro), were the lowest used antidiabetics as they were recently introduced to the Saudi pharmaceutical health market and require some time to be commonly prescribed for the patients with T2DM. In addition, they are more expensive than the other remaining antidiabetic classes. GLP-1 analogs such as Liraglutide (Victoza) is a non-insulin antidiabetic which is a cardiovascular protective that 
reduces cardiovascular events such as heart attack, stroke, or death ${ }^{11}$ and could replace the use of both Metformin and DPP-4 inhibitors with better glycemic control and in the long run especially for patients with obesity. ${ }^{30,31}$ Therefore, it is highly recommended for Saudi patients with T2DM to use GLP-1 analogs to manage T2DM better to reduce the risk of associated complications.

It is important to note that participants who demonstrated good knowledge may not necessarily lead to a good attitude and practice. That is because even though they knew the benefits of exercise and the importance of eating food less in carbohydrates and lipids, many of them did not comply and followed a planned diet regimen or an exercise program to maintain their BGLs and improve daily T2DM practice. However, this may be due to several factors that can be modifiable (self-efficacy, time management, pharmaceutical intervention and drug compliance, psychological factors such as motivation and depression) or non-modifiable (age, sex, and race). Therefore, developing innovative tools and educational models to improve patients' attitudes and practice; hence their self-awareness, is directly associated with improving the patient's compliance that reduced T2DM complications. ${ }^{9,10}$

According to the systemic review conducted by Meo, ${ }^{32}$ which stated that the prevalence of T2DM in SA has the highest prevalence $(32.8 \%)$ in 2015 . They expected the prevalence to increase from $32.8 \%$ in 2015 to $45.36 \%$ by 2030 . Another study estimated that $13.9 \%$ of SA's health budget was expended towards T2DM management. ${ }^{33}$ Therefore, it is crucial to improve the quality of life to reduce disease costs and burdens ${ }^{32,33}$ Clinical pharmacists can use the results of this KAP study and other similar studies in SA to establish an improved clinical and pharmaceutical comprehensive health education program that contributes to and enhances the patient's self-management, drug awareness, and compliance, and daily practice for patients with T2DM living in Makkah region, SA for better management of T2DM complications and reduce the patient's hospital emergencies referrals. ${ }^{14,34-37}$

\section{Conclusion}

Our results showed that patients with T2DM in the Makkah region showed good responses regarding T2DM knowledge; however, this was not reflected in their poor attitude and practice levels. Therefore, self-management among patients with T2DM in Makkah region, especially in the Holy Capital, must be increased to avoid potential health difficulties in diabetes health clinics during Hajj and Umrah seasons. In addition, it is crucial to enhance the use of recently introduced antidiabetic medication, especially GLP-1 analogous, which are cardiovascular protective, provide better glycemic control, and help manage associated complications among patients with T2DM. Furthermore, clinical pharmacists can offer comprehensive clinical pharmaceutical education programs to enhance the knowledge and awareness of T2DM, related symptoms, and complications, and deliver the most recent approved antidiabetics classes used for the treatment of T2DM towards the patients and health providers to improve their self-management of T2DM, antidiabetic pharmaceutical knowledge, and their compliance. Furthermore, patients with T2DM are highly advised to visit the diabetes health care centers and enroll in clinical pharmaceutical education programs to enhance their KAP levels to improve their self-management and drug compliance. Due to the increased prevalence of T2DM in SA, there is an urgent implementation of improved clinical pharmaceutical, educational programs for those with T2DM. This is to avoid T2DM complications and reduce morbidity and mortality, such as; early detection of the disease and improving patient's self-management, which is costeffective in reducing the disease's cost and burden. ${ }^{32,33}$

\section{Study limitations}

The study was conducted in the Makkah region, SA; therefore, it may not necessarily reflect the actual number of the general populations among all SA regions. Also, the sample size is not representative of the studied region. The total number of the Makkah region is estimated to be more than 4.5 million people $;{ }^{18}$ however, it still can be considered a statistically representative random sample. In addition, the prevalence of only the major six antidiabetic classes was measured among the patients, and the prevalence of the remaining medication and natural (herbal) products that could affect the glycaemic control was not estimated. Moreover, fasting blood glucose (FBG) and Haemoglobin A1C (HbA1C) measurement were not studied. This, however, can be useful to study the correlation between these measurements and KAP of T2DM, which could reflect the effect of knowledge and attitude on the patient's glycaemic control.

\section{Acknowledgments}

Special thanks to the dedicated senior pharmacy students who contributed to this clinical research and data collecting which contributed to the successful completion of this study; Mr. Mohammad H. Alsaedi, Ms. Alya'a B. Bannani, Mr. Motlaq N. Al-Shammari, Mr. Torki A. Alzahrani, Mr. Mansur J. Al-Otaibi, Ms. Shahd Y. Alharbi, Mr. Mohammed H. Alharthy, and Mr. Anmar A. Alrefaei. Special thanks to KAMC for approving and supporting this observational study and facilitating our data collectors to approach potential patients in Makkah region health clusters. Many thanks to the medical staff in governmental hospitals of the health clusters and sales managers in the Makkah region's leisure centers and shopping malls for their unlimited help and support during the data collection period. Many thanks to all the participants who dedicated the time to complete this questionnaire of this study voluntarily.

\section{Disclosure}

The authors declare no conflicts of interest in this study.

\section{References}

1. Blair M. Diabetes Mellitus Review. Urol Nurs. 2016;36(1):27-36.

2. Roper NA, Bilous RW, Kelly WF, et al. Cause-specific mortality in a population with diabetes: South Tees Diabetes Mortality Study. Diabetes Care. 2002;25(1):43-48.

3. IDF DIABETES ATLAS, 9th edition 2019. International Diabetes Federation; 2019.

4. Health Statistics Report, Health SMo. Ministry of Health, Saudi Arabia; 2018 .

5. Rawal LB, Tapp RJ, Williams ED, et al. Prevention of type 2 diabetes and its complications in developing countries: a review. Int J Behav Med. 2012;19(2):121-133.

6. MOH MoHiSA. Endocrine Disease, Diabetes 2019.

7. NIDDK TNIoDaDaKD. Health Information about Diabetes; 2019.

8. Diabetes, Key Facts. The World Health Orgnization Website; 2019.

9. Shrivastava SR, Shrivastava PS, Ramasamy J. Role of self-care in management of diabetes mellitus. J Diabetes Metab Disord. 2013;12(1):14.

10. Gunggu A, Thon CC, Whye Lian C. Predictors of Diabetes SelfManagement among Type 2 Diabetes Patients. $J$ Diabetes Res. 2016;2016:9158943.

11. Victoza NN. Victoza Liraglutide 1.2, 1.8 mg: Novo Nordisk; 2021.

12. Kovacich N, Chavez B. Ertugliflozin (Steglatro): A New Option for SGLT2 Inhibition. P T. 2018;43(12):736-742. 
13. Ministry of Hajj and Umrah SA. Hajj \& Umrah Services 2021.

14. A guide to developing knowledge attitude and practice surveys. World Health Organization, Stop TB Partnership: WHO; 2008. 68 p.

15. Ibrahim Abougalambou SS, AbaAlkhail H, Abougalambou AS. The knowledge, attitude and practice among diabetic patient in central region of Saudi Arabia. Diabetes Metab Syndr. 2019;13(5):2975-2981.

16. Alqahtani M, Almutairi FE, Albasseet AO, et al. Knowledge, Attitude, and Practice of Diabetes Mellitus Among the Saudi Population in Riyadh, Saudi Arabia: A Quantitative Study. Cureus. 2020;12(1):e6601.

17. Aljofan M, Altebainawi A, Alrashidi MN. Public knowledge, attitude and practice toward diabetes mellitus in Hail region, Saudi Arabia. Int J Gen Med. 2019;12:255-262.

18. General Authority for Statisics SA. Population in Makkah region by gender, age group, and nationality (Saudi/Non-Saudi). General Authority for Statisics: Saudi Arabia; 2017.

19. Monkey S. Sample Size Calculator. Survey Monkey website.

20. MDRTC TMDRaTC. Tools for Health Professionals, Survey Instruments University of Michigan Health System website; 2012.

21. Ghannadi S, Amouzegar A, Amiri P, et al. Evaluating the Effect of Knowledge, Attitude, and Practice on Self-Management in Type 2 Diabetic Patients on Dialysis. Journal of Diabetes Research. 2016;2016:3730875.

22. Ahmed MU, Seriwala HM, Danish SH, et al. Knowledge, Attitude, and Self Care Practices Amongsts Patients WithType 2 Diabetes in Pakistan. Glob J Health Sci. 2015;8(7):1-8.

23. Saleh F, Afnan F, Ara F, et al. Diabetes Education, Knowledge Improvement, Attitudes and Self-Care Activities Among Patients With Type 2 Diabetes in Bangladesh. Jundishapur J Health Sci. 2017;9(1):e36058.

24. Niguse H, Belay G, Fisseha G, et al. Self-care related knowledge, attitude, practice and associated factors among patients with diabetes in Ayder Comprehensive Specialized Hospital, North Ethiopia. BMC Research Notes. 2019;12(1):34

25. Suppapitiporn S, Chindavijak B, Onsanit S. Effect of diabetes drug counseling by pharmacist, diabetic disease booklet, and special medication containers on glycemic control of type 2 diabetes mellitus: a randomized controlled trial. J Med Assoc Thai. 2005;88 Suppl 4:S134-S141.
26. Association AD. Standards of Medical Care in Diabetes-2007. Diabetes Care. 2007;30(Suppl 1):S4-S41.

27. Alqahtani M, Almutairi FE, Albasseet AO, et al. Knowledge, Attitude, and Practice of Diabetes Mellitus Among the Saudi Population in Riyadh, Saudi Arabia: A Quantitative Study. Cureus. 2020;12(1):e6601.

28. Latif Ahmed Khan, Khan SA. Level of Knowledge and Self-Care in Diabetics in a Community Hospital in Najran. Annals of Saudi Medicine. 2000;20(3-4):300-301.

29. AlKhamees OA, AlNemer KA, Bin Maneea MW, et al. Top 10 most used drugs in the Kingdom of Saudi Arabia 2010-2015. Saudi Pharm J. 2018;26(2):211-216.

30. Ponzani P. Long-term effectiveness and safety of liraglutide in clinical practice. Minerva Endocrinol. 2013;38(1):103-112.

31. Ponzani P, Corsi A. Incretin-based therapies in clinical practice: from efficacy to effectiveness. Focus on liraglutide. Minerva Endocrinol. 2012;37(1):1-8.

32. Meo SA. Prevalence and future prediction of type 2 diabetes mellitus in the Kingdom of Saudi Arabia: A systematic review of published studies. $J$ Pak Med Assoc. 2016;66(6):722-725.

33. Robert AA, Al Dawish MA, Braham R, et al. Type 2 Diabetes Mellitus in Saudi Arabia: Major Challenges and Possible Solutions. Curr Diabetes Rev. 2017;13(1):59-64

34. Askari A, Jeihooni AK, Kashfi SM, et al. The Effect of Educational Program Based on Belief, Attitude, Subjective Norm, and Enabling Factors Model on Changing the Metabolic Indices in Elderly Patients with Type II Diabetes. Int J Prev Med. 2018;9:74.

35. Chawla SPS, Kaur S, Bharti A, et al. Impact of health education on knowledge, attitude, practices and glycemic control in type 2 diabetes mellitus. J Family Med Prim Care. 2019;8(1):261-268.

36. Ismaile S, Alhosban F, Almoajel A, et al. Knowledge, Attitude and Practice Tools for Health Education among Diabetic Patients. Stud Health Technol Inform. 2017;238:250-252.

37. Wang H, Song Z, Ba Y, et al. Nutritional and eating education improves knowledge and practice of patients with type 2 diabetes concerning dietary intake and blood glucose control in an outlying city of China Public Health Nutr. 2014;17(10):2351-2358. 